\title{
Gender discrimination, victimisation and women's mental health
}

Georgina M. Hosang and Kamaldeep Bhui

\section{Summary}

Gender inequality and discrimination, as well as violence and victimisation towards women, have recently hit the headlines creating a media furore. We provide a timely discussion surrounding the impact of these issues on women's mental health and a discussion of the role of psychiatry in this context.

\section{Declaration of interest}

K.B. is the editor for the British Journal of Psychiatry but has not played a role in the decision to accept this editorial for publication in this journal. G.H. has no conflict of interest to declare.

\section{Keywords}

Women's mental health; gender discrimination; victimisation; childhood maltreatment.

\section{Copyright and usage}

(c) The Royal College of Psychiatrists 2018.
Georgina Hosang (pictured) is a senior lecturer researching the impact of psychosocial stress and its interplay with genetic factors on mood disorders and physical illnesses; Kamaldeep Bhui is a psychiatrist and professor interested in the sociocultural risk and protective factors related to inequalities in population mental health.

Mental health problems affect men and women equally ${ }^{1}$ but a female preponderance is observed in some illnesses, such as major depression and anxiety disorders. ${ }^{2}$ Moreover, the burden of mental disorders is greater in females compared to males according to disability-adjusted life years. ${ }^{3}$ Explanations for these gender patterns have asserted biological causation due to hormonal differences, vulnerability to female-specific medical illnesses (e.g. ovarian cancer), reproductive risks of physical illness and puerperal psychiatric disorders (e.g. postnatal depression). Disruption or abnormalities in some of these biological factors (i.e. reproductive health) can directly impact on women's mental health, best illustrated with Premenstrual Dysphoric Disorder. Women are also at greater risk of experiencing various psychosocial stressors (e.g. sexual violence) that are key determinants of poor physical and mental health outcomes, ${ }^{4}$ but are preventable. Gender discrimination, also referred to as gender disadvantage, inequalities or disparities encompass a range of experiences and structural inequalities. These include the increased likelihood that women may hold positions of lower power in work settings and lower status in societies in which women are not protected against violence or are discouraged from pursuing an education and independent living. ${ }^{5}$ The negative impact of gender discrimination on physical and mental health can be compounded by its 'correlates' (e.g. poverty). These factors can cause much stress and strain on women as well as impact their opportunity to access health care.

Here we will focus on gender discrimination and victimisation since they have received less attention in the women's mental health literature compared to biological factors. The prevalence of gender discrimination in the workplace, such as sexual harassment and gender pay inequalities, have recently been publicly illuminated with the \#MeToo and \#TimesUp movements and campaigns championed by both Hollywood stars and activists such as Tarana Burke. The public and media interest in gender discrimination and victimisation towards women forces us to stop and review the pertinence of these issues on women's mental health.

\section{Does gender discrimination affect women's mental health?}

Sex is a biological fact based on physiological characteristics, whereas gender is a socio-cultural construct that encompasses norms and attitudes related to social roles, statuses and abilities. Discrimination exists when these social norms and attitudes stipulate that women should hold a subordinate position. As a result, women may hold a 'minority' status based on their gender within a society or subculture where a gender power imbalance exists, despite females representing approximately half of the population.

Gender discrimination impacts all aspects of a woman's life, such as limited access to employment opportunities and quality health care, which can result in poor health outcomes. This power imbalance becomes especially troubling in resource-poor areas where multiple disadvantages cluster. In addition to discrimination, women who do not conform to the socio-cultural norms of their gender can face stigma, social exclusion and violence (e.g. 'honour killings'). Women can hold multiple forms of minority statuses (e.g. due to their ethnicity), meaning that they can be subject to discrimination from a myriad of angles, which together leads to greater stress and worse mental health outcomes. There is growing recognition that gender-influenced isolation, social exclusion, bullying and threats all add to the burden and injustice of discrimination. Yet there is a dearth of research that explores gender disadvantage and women's mental health. This discourse should not undermine resilience, fortitude and that action is taking place, but should be taken as an opportunity to raise the profile of these issues.

Discrimination can be subtle or explicit, conscious or unconscious and permeates into all life domains and areas of society. Gender discrimination in the workplace includes harassment, unequal pay and implementation of rules that puts one gender at a disadvantage. Although gender discrimination is illegal in many countries it is still evident. For instance, statistics from Europe show that women earn on average $20 \%$ less than men. ${ }^{6}$ In the UK, over $50 \%$ of women surveyed reported experiencing sexual harassment in the workplace (e.g. unwanted sexual touching). ${ }^{7}$ Experimental evidence shows gender-biased hiring preferences where men are favoured over women (even though qualifications and experience are identical); interestingly, both men and women exhibited these biases. $^{8}$

Although instances of gender discrimination are still common, the cultures in which these incidents happen are usually structured 
to not notice or prevent such discrimination. For example, gender power imbalances exist in policies and processes (e.g., primogeniture, law or custom pertaining to inheritance of firstborn son which overlooks daughters), reinforcing a certain mindset that supports or encourages gender discrimination. The power of group conformity and dependency makes it particularly challenging to speak out against instances of discrimination and requires courage and strength to stand up to the institution and individuals. Responses to such action include avoiding acknowledgement and responsibility, making reporting and addressing discrimination difficult.

Perceived gender discrimination has been associated with various psychiatric disorders, including major depression and post-traumatic stress disorder [PTSD]. ${ }^{9}$ Research shows that the experience of gender discrimination accounts for more of the variance for depressive, anxiety and somatic symptoms in women compared with psychosocial stressors (known predictors of depression). ${ }^{10}$ Similar patterns are observed when more objective measures of discrimination are considered (e.g. gender pay inequalities). Women who earn less than men have significantly higher odds of major depression than their male counterparts. ${ }^{11}$ However, no significant gender differences in depression are observed among matched pairs in which women had a higher income than men. ${ }^{11}$ Interestingly, the female preponderance in depression has been found to be less pronounced in recent years and has been attributed to changes in the traditionality of female gender roles (e.g. better female education). ${ }^{5}$ These findings provide preliminary but convincing evidence that gender discrimination plays a prominent role in women's mental health.

Greater efforts are needed to reduce gender discrimination throughout society by raising awareness, development of new and better implementation of existing policies, more equal representation of females in positions of power, as well as structures and environments that promote gender equality.

\section{Does victimisation have a negative impact on women's mental health?}

Physical violence, sexual assaults, and psychological, economic and emotional abuses can all be categorized under the construct of victimisation. Depending on the age of the victim and the perpetrator these types of victimisation can be classified as childhood maltreatment, where the victim is a child or adolescent, and includes physical, sexual, psychological and/or emotional abuse as well as neglect (lack of provision for the individual's physical and emotional needs by their caregiver) perpetrated by an adult (or an older child for sexual abuse). Domestic or intimate partner violence and abuse is defined as one partner using physical, sexual, financial and/or emotional abuse to gain or maintain control or power over their partner. Females are at greater risk of being exposed to childhood maltreatment ${ }^{12}$ and domestic violence and abuse. ${ }^{4}$ Gender victimisation also includes forced marriage, 'honour' crimes and human trafficking, ${ }^{4}$ however, a review of their impact on women's mental health is outside the scope of this discussion.

Childhood maltreatment is significantly associated with physical and mental illnesses, ${ }^{13}$ such as cardiovascular disease and mood disorders in adulthood; these associations have been shown to be strongest in women. ${ }^{14,15}$ The experience of domestic violence and abuse can lead to the development of mental health problems. For instance, a recent meta-analysis reported a seven-times increase in the likelihood of PTSD among women exposed to domestic violence and abuse. ${ }^{16}$ Childhood maltreatment is also a risk factor for domestic violence and abuse. ${ }^{17}$ In fact, victimisation in childhood and adulthood has been shown to have an accumulative impact on mental health outcomes in a dose-response fashion. Specifically, women with a history of childhood maltreatment, and who are exposed to domestic violence and abuse, have the highest risk of depression followed by those who have experienced only one of these victimisation types; women with no such victimisation histories show the lowest risk of depression. ${ }^{17}$ In sum, the research evidence converges to show the significant independent and combined impact of childhood maltreatment and domestic violence and abuse on women's mental health. Recommendations for mental health services based on such findings include the implementation of gender and trauma sensitive protocols regarding clinical assessment and access to trauma-informed interventions as well as staff training in these areas.

\section{Gender inequality and women's mental health, what is psychiatry doing about it?}

As a clinical and academic community, psychiatry has a responsibility to address gender disadvantage to improve the mental health of its female staff and patients. Female psychiatrists are underrepresented in senior roles in academia, for instance approximately $20 \%$ of UK clinical professors are female. ${ }^{18}$ This is disappointing and highlights the need for action. However, this figure is also encouraging since it is similar to the proportion of female non-clinical lecturers (29\%) and is higher than the 2007 figures where only $13 \%$ of clinical professors were women. ${ }^{19,20}$ In terms of leadership, both the President and the Dean of the UK's Royal College of Psychiatrists (RCPsych) are women. Other societies for professionals that work in mental health research and care also have female presidents (e.g. the British Psychological Society and the Royal College of Nurses).

Increasing academic dialogue concerned with gender disadvantage and women's mental health has been sparked by initiatives, including taskforces and special interest groups within the RCPsych and the World Psychiatric Association (WPA). The Lancet has also published a series of articles focused on 'advancing women in science, medicine and global health'. It is hoped that these efforts will translate into real change in intellectual thinking, research and institutional structures, ultimately resulting in gender equality.

Women are overrepresented in the delivery and management of mental health care, making up to $80 \%$ of those that provide and $65 \%$ that manage psychological services in the NHS. ${ }^{21}$ However, men represent a higher proportion of those that shape and influence such services (e.g. commissioners). ${ }^{21}$ Greater efforts to achieve gender balance across all levels of NHS mental health services are needed to better reflect the population it serves.

Steps have been taken to account for the impact of gender disadvantage and violence clinically. For example, the WPA has produced a training curriculum for mental health care providers on intimate partner violence and sexual violence against women. Some of the principles of this training are in line with the trauma-informed mental health care (TIMHC) framework (i.e. recognition) that has seen calls for implementation in the $\mathrm{UK}^{22}$ The rationale behind the need for TIMHC is based on two points: first, that a considerable portion of people with mental health problems have experienced trauma; and second, mental health care can involve coercion and control (e.g. restraint and forced medication) which can trigger similar emotional, cognitive and physiological responses associated with previous traumatic experiences and thus may re-traumatise survivors. TIMHC focuses on building trust between service users and providers and creating safe therapeutic environments, based on principles such as transparency and survivor partnerships. ${ }^{22}$ The beneficial impact of TIMHC includes the reduction in the number of seclusions/restraints and time to discharge, ${ }^{22}$ and has been included in the NHS England's strategic plan for sexual assault and abuse services. 


\section{Summary and conclusions}

Women are exposed to a plethora of adverse experiences and environments that negatively impact on their health and wellbeing. Many of these factors are either gender-specific (e.g. gender discrimination) or more common in women (e.g. childhood maltreatment). ${ }^{4,12}$ These factors intersect, with many women experiencing multiple factors at the same time or across their lifetime, creating a lethal cocktail of risk that results in poor health, social and economic outcomes. There is an overwhelming need for co-ordinated prevention and intervention efforts that tackle these issues from societal, community and individual levels.

Gender-specific and trauma-sensitive strategies are recommended by the WHO and the UK's National Institute of Health and Care Excellence. ${ }^{4}$ These can manifest as staff training, administration of clinical assessments without women's partners or family members, and/or the use of independent interpreters (if needed) rather than reliance on family or friends. ${ }^{4}$ As a clinical and academic community we have a responsibility to raise awareness of these issues, ensuring that our structures and policies create a working environment and service that promotes gender equality and women's mental health.

Georgina M. Hosang (iD), Senior Lecturer, Centre for Psychiatry, Wolfson Institute of Preventive Medicine, Barts \& The London School of Medicine \& Dentistry, Queen Mary, University of London, UK; Kamaldeep Bhui (iD, Centre for Psychiatry, Wolfson Institute of Preventive Medicine, Barts \& The London School of Medicine \& Dentistry, Queen Mary, University of London, UK

Correspondence: Dr Georgina Hosang, Centre for Psychiatry, Wolfson Institute of Preventive Medicine, Barts \& The London School of Medicine \& Dentistry, Queen Mary, University of London, Old Anatomy Building, Charterhouse Square, London EC1M 6BQ, UK. Email: g.hosang@qmul.ac.uk

First received 13 Apr 2018, final revision 5 Sep 2018, accepted 10 Oct 2018

\section{References}

1 Kessler RC, Berglund P, Demler O, Jin R, Merikangas KR, Walters EE. Lifetime prevalence and age-of-onset distributions of DSM-IV disorders in the National Comorbidity Survey Replication. Arch Gen Psychiatry 2005; 62: 593-602.

2 Seedat S, Scott KM, Angermeyer MC, Berglund P, Bromet EJ, Brugha TS, et al. Cross-national associations between gender and mental disorders in the WHO World Mental Health Surveys. Arch Gen Psychiatry 2009; 66: 785-95.

3 Whiteford HA, Degenhardt L, Rehm J, Baxter AJ, Ferrari AJ, Erskine HE, et al. Global burden of disease attributable to mental and substance use disorders: findings from the Global Burden of Disease Study 2010. Lancet 2013; 382 $1575-86$

4 Oram S, Khalifeh $\mathrm{H}$, Howard LM. Violence against women and mental health. The Lancet Psychiatry 2017; 4: 159-70.

5 Seedat S, Scott KM, Angermeyer MC, Berglund P, Bromet EJ, Brugha TS, et al. Cross-national associations between gender and mental disorders in the WHO World Mental Health Surveys. Arch Gen Psychiatry 2009; 66: 785-95.

6 EIGE. Gender Equality Index 2015. Measuring gender equality in the European Union 2005-2012. 2015. doi:10.2839/763764.

7 TUC and Everyday Sexism Project. Still just a bit of banter? 2016.

8 Moss-Racusin CA, Dovidio JF, Brescoll VL, Graham MJ, Handelsman J. Science faculty's subtle gender biases favor male students. Proc Natl Acad Sci 2012; 109: 16474-9.

9 McLaughlin KA, Hatzenbuehler ML, Keyes KM. Responses to discrimination and psychiatric disorders among black, hispanic, female, and lesbian, gay, and bisexual individuals. Am J Public Health 2010; 100: 1477-84.

10 Klonoff EA, Landrine $H$, Campbell R. Sexist discrimination may account for well-known gender differences in psychiatric symptoms. Psychol Women $Q$ 2000; 24: 93-9.

11 Platt JM, Prins SJ, Bates LM, Keyes KM. Erratum: Corrigendum to ‘Unequal depression for equal work? How the wage gap explains gendered disparities in mood disorders' (Soc. Sci. Med. (2016) (1-8). Soc Sci Med 2016; 162: 229.

12 Christoffersen MN. The prevalence of four types of childhood maltreatment in Denmark. Clin Pract Epidemiol Ment Heal 2013; 9: 149-56.

13 Hosang GM, Fisher HL, Uher R, Cohen-Woods S, Maughan B, McGuffin P, et al. Childhood maltreatment and the medical morbidity in bipolar disorder: a casecontrol study. Int J Bipolar Disord 2017; 5: 30.

14 Hosang GM, Johnson SL, Kiecolt-Glaser J, Di Gregorio MP, Lambert DR, Bechtel MA, et al. Gender specific association of child abuse and adult cardiovascular disease in a sample of patients with Basal Cell Carcinoma. Child Abus Neg/ 2013; 37, doi:10.1016/j.chiabu.2012.09.018.

15 Gallo EAG, Munhoz TN, Loret de Mola C, Murray J. Gender differences in the effects of childhood maltreatment on adult depression and anxiety: a systematic review and meta-analysis. Child Abus Neg/ 2018; 79: 107-14.

16 Trevillion K, Oram S, Feder G, Howard LM. Experiences of domestic violence and mental disorders: a systematic review and meta-analysis. PLOS One 2012; 7: e51740.

17 Ouellet-Morin I, Fisher HL, York-Smith M, Fincham-Campbell S, Moffitt TE, Arseneault L. Intimate partner violence and new-onset depression: a longitudinal study of Women's childhood and adult histories of abuse. Depress Anxiety 2015; 32: 316-24.

18 Watson N, Tang P, Knight E. Survey of Medical Clinical Academic Staffing Levels. 2018.

19 Medical Schools Council. Women in Clinical Academia. 2007

20 HESA. Staff in Higher Education 2016/2017. 2018.

21 Morison L, Trigeorgis C, John M. Are mental health services inherently feminised? Psychologist 2014; 27 : 414-6.

22 Sweeney A, Clement S, Filson B, Kennedy A. Trauma-informed mental healthcare in the UK: what is it and how can we further its development? Ment Heal Rev J 2016; 21: 174-92. 